\title{
EFECTO DEL EXTRACTO ACUOSO DE LA PAPA NATIVA Solanum tuberosum "PUCA SIMI" SOBRE ENZIMAS DE DETOXIFICACIÓN DE FASE II
}

\author{
Effect of aqueous extract of native potato Solanum tuberosum "puca simi" on enzymes of \\ phase II detoxification
}

Emilio Ramírez ${ }^{1}$, Silvia Suárez ${ }^{2}$

${ }^{1}$ Escuela de Formación Profesional de Farmacia y Bioquímica, Universidad Nacional de San Cristóbal de Huamanga. ${ }^{2}$ Centro de Investigación de Bioquímica y Nutrición, Facultad de Medicina Humana. Universidad Nacional Mayor de San Marcos.

\section{RESUMEN}

El objetivo del presente estudio fue evaluar el efecto del extracto acuoso de la papa nativa Solanum tuberosum "puca simi" sobre enzimas de detoxificación de fase II. Se emplearon 28 ratas macho adultas de cepa Rattus norvegicus de 200 a $250 \mathrm{~g}$ de peso, en cuatro grupos de siete cada uno, a los que se les administró porvía orogástrica: Grupo I control, suero fisiológico; Grupo II, fenilhidrazina $60 \mathrm{mg} / \mathrm{kg}$ de peso; Grupo III, extracto acuoso de papa $665 \mathrm{mg} / \mathrm{kg}$ de peso; Grupo IV, extracto acuoso de papa $665 \mathrm{mg} / \mathrm{kg}$ y fenilhidrazina $60 \mathrm{mg} / \mathrm{kg}$. Se determinaron los niveles de proteínas totales, bilirrubina total, malondialdehido (MDA), hematocrito y las actividades de glutation S-transferasa (GST), Uridin difosfato-glucuroniltransferasa (UDP-GT). En el estudio fitoquímico se identificaron polifenoles, flavonoides y saponinas. Se encontró una disminución significativa de los niveles de hematocrito, bilirrubina total y proteínas totales en los grupos III y IV respecto al control y grupo II, asimismo una disminución significativa $(p<0,05)$ del malondialdehido en el grupo III $(1,53 \mathrm{gg} / \mathrm{g}$ tejido) y IV $(1,56 \mathrm{gg} / \mathrm{g}$ tejido $)$ respecto al grupo II (2,03 $\mathrm{gg} / \mathrm{g}$ tejido). La actividad específica de la UDP-glucuroniltransferasa aumentó significativamente $(p<0,01)$ en el grupo IV respecto al control y los demás grupos, mientras la actividad específica y total de la glutatión S-transferasa no exhibe ninguna diferencia significativa en los cuatro grupos. Se concluye que el extracto acuoso de papa nativa "puca simi" contiene metabolitos secundarios que protegen la lipoperoxidación e incrementa de manera significativa la actividad de la UDP-glucuroniltransferasa.

Palabras clave: Papa nativa "puca simi", lipoperoxidación, UDP-glucoroniltransferasa, glutation S-transferasa, metabolismo de fase II.

\section{SUMMARY}

The objective of this study was to evaluate the effect of aqueous extract of native potato Solanum tuberosum "puca simi " on detoxification enzymes of phase II. Were used 28 male rats Rattus norvegicus strain of 200 to $250 \mathrm{~g}$, that were grouped into four groups of seven each, receiving by orogastric way: Group I control, saline; Group II, phenylhydrazine 6o $\mathrm{mg} / \mathrm{kg}$; Group III, potato extract $665 \mathrm{mg} / \mathrm{kg}$; Group IV, potato extract and phenylhydrazine. Were determined levels of total protein, total bilirubin, malondialdehyde (MDA), hematocrit; the activities of glutathione S-transferase (GST) and UDPglucuronyltransferase. The phytochemical study identified polyphenols, flavonoids and saponins. A significant decrease in hematocrit levels, total bilirubin, and total protein in groups III and IV regarding the control and group II was found, also a significant $(p<0,05)$ malondialdehyde in group III $(1,53 \mathrm{gg} / \mathrm{g}$ tissue $)$ and IV $(1,56 \mathrm{gg} / \mathrm{g}$ tissue $)$ compared to group II $(2,03$ $\mathrm{jg} / \mathrm{g}$ tissue). The specific activity of UDP-glucuronyl transferase increased significantly $(p<0,01)$ in group IV compared to the control and other groups, and the specific and total activity of glutathione S-transferase exhibits no significant difference in the four groups. It is concluded that the aqueous extract of native potato "pucca simi" contains secondary metabolites that protect lipid peroxidation and significantly increases the activity of the UDP-glucuronosyltransferase.

Keywords: Native potato "puca simi", lipoperoxidation, UDP-glucuronyltransferase, glutathione S-transferase, phase II metabolism.

\section{INTRODUCCIÓN}

as papas nativas constituyen productos con un potencial nutricional, nutracéutico y comercial interesante; se cultivan sobre los 3000 metros de altitud en las comunidades de la región andina de nuestro país que viven en pobreza o en pobreza extrema ${ }^{(1)}$ y contienen metabolitos secundarios con diversas propiedades medicinales, como los polifenoles ${ }^{(2)}$. Más allá de las propiedades antioxidantes, 
Tabla 1. Marcha fitoquímica del extracto acuoso de la papa nativa Solanum tuberosum "puca simi".

\begin{tabular}{cccc}
\hline Metabolitos secundarios & Reacción & Resultados & Observaciones \\
\hline Taninos & Gelatina & -- & Negativo \\
Aminoácidos libres & Ninhidrina & -- & Negativo \\
Flavonoides & Shinoda & ++ & Coloración roja \\
Compuestos fenólicos & $\mathrm{FeCl}_{3}$ & ++ & Coloración verde \\
Saponinas & Ensayo de espuma & ++ & Abundante espuma \\
Triterpenos y/o esteroides & Liebermann-Burchard & -- & Negativo \\
\hline
\end{tabular}

$(-)$ : No se evidencia presencia, (+): Presencia de trazas, (++): Presencia moderada, (+++): Presencia abundante

En la actualidad existen estudios experimentales de la actividad hepatoprotectora de varios recursos terapéuticos, que pueden ser considerados como terapias alternativas para el tratamiento de las enfermedades hepáticas y sus complicaciones ${ }^{(5)}$.

La investigación del extracto a estos compuestos se les atribuye varias acciones terapéuticas, como cardioprotectores, hepatoprotectores, anticancerígenos y neuroprotectores ${ }^{(3)}$.

El hígado es el principal órgano del metabolismo y) está sujeto a una elevada incidencia de enfermedades, que en el Perú, ocupan el quinto lugar como causa de mortalidad y el segundo como causa de morbilidad ${ }^{(4)}$.

acuoso de la "puca simi" como un hepatoprotector, formaría parte de las terapias alternativas para estos padecimientos. Por ello, los objetivos del estudio fueron evaluar el efecto del extracto acuoso de la papa nativa Solanum tuberosum "puca simi" sobre enzimas de detoxificación de fase II, la peroxidación lipídica, los niveles de bilirrubina total sérica, proteínas totales,

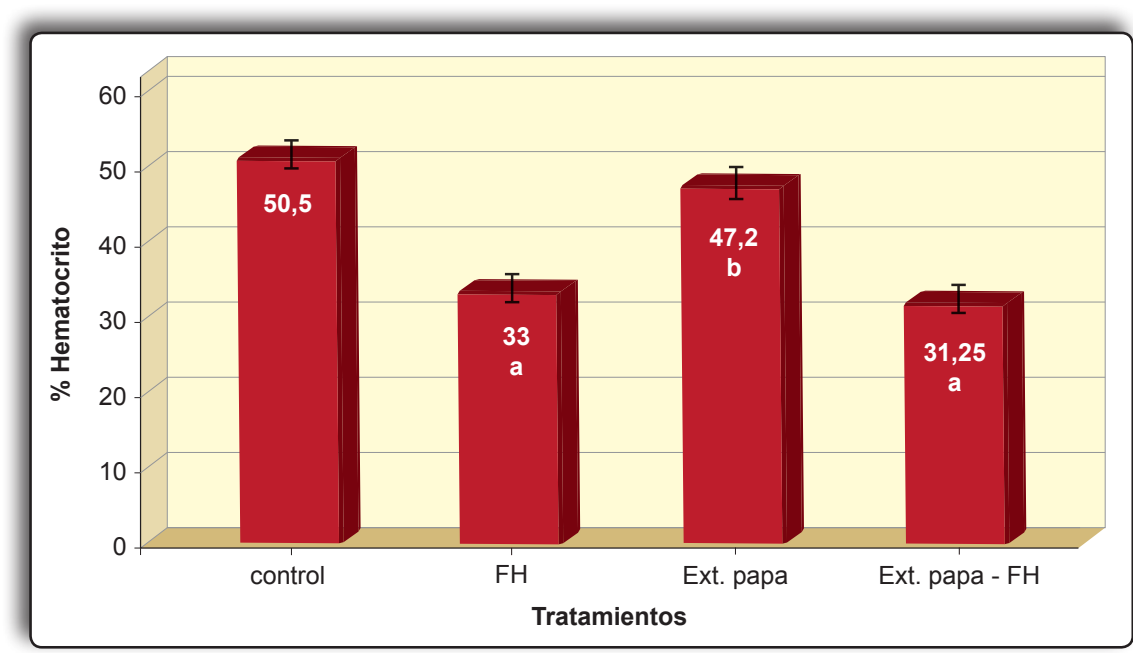

Figura 1. Hiperbilirrubinemia por inducción de anemia hemolítica expresada en Hematocrito. Las diferencias significativas $(p<0,05)$ están expresadas con la letra (a) con respecto al grupo control y con la letra (b) con respecto al grupo FH.

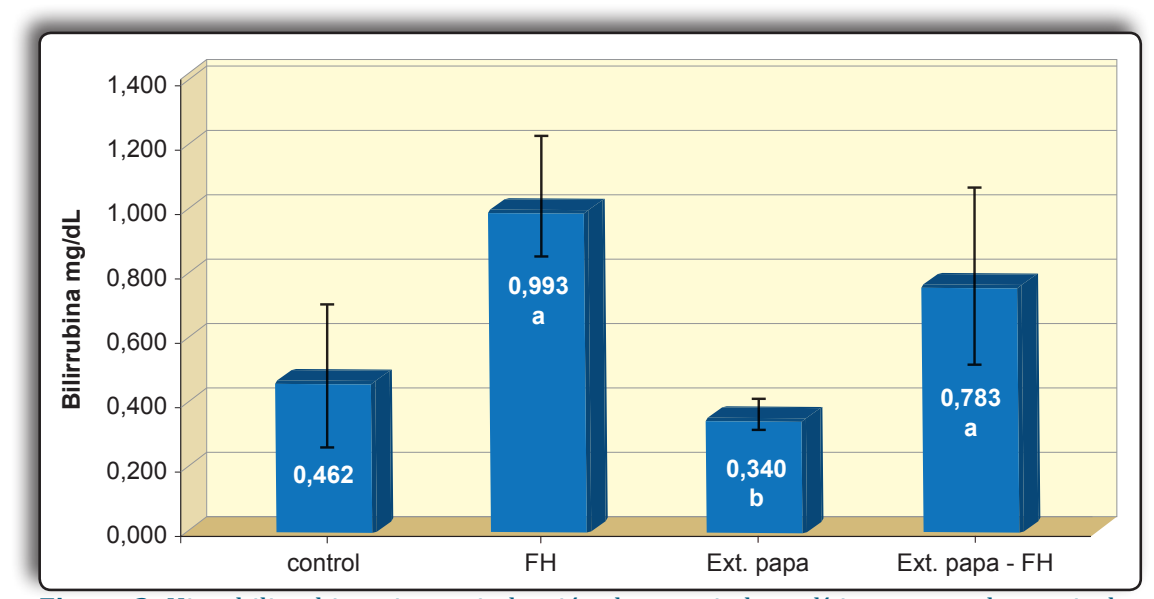

Figura 2. Hiperbilirrubinemia por inducción de anemia hemolítica expresada en niveles plasmáticos de bilirrubina total. Las diferencias significativas contra el control son expresadas como (a) y las diferencias contra FH son expresadas como (b), $(p<0,05)$. hematocrito; e identificar los metabolitos secundarios.

\section{MATERIALES Y MÉTODOS}

Las papas nativas fueron recolectadas en la comunidad de Champaccocha del distrito de San Jerónimo, provincia de Andahuaylas, Región Apurímac, ubicada a 3500 metros de altitud. La muestra fue cortada y secada en estufa entre 38 $40^{\circ} \mathrm{C}$ con circulación permanente de aire, finalmente fueron molidas hasta obtener un polvo fino. Se preparó un macerado al $10 \%$ con agua bidestilada acidulada por $24 \mathrm{~h}$ en baño maría a $35^{\circ} \mathrm{C}$ con agitación constante. El extracto se centrifugó a 7000 rpm por 20 minutos, luego se evaporó la solución a $40^{\circ} \mathrm{C}$ hasta obtener el extracto seco y se realizó la marcha fitoquímica para la identificación de metabolitos secundarios.

Se emplearon 28 ratas machos adultos de cepa Rattus norvegicus de 200 a $250 \mathrm{~g}$ de peso adquiridas en el Instituto Nacional de SaludChorrillos, las que fueron alojadas y aclimatadas durante una semana antes del experimento en el bioterio del Centro de Investigación de Bioquímica y Nutrición de la Facultad de Medicina, a una 


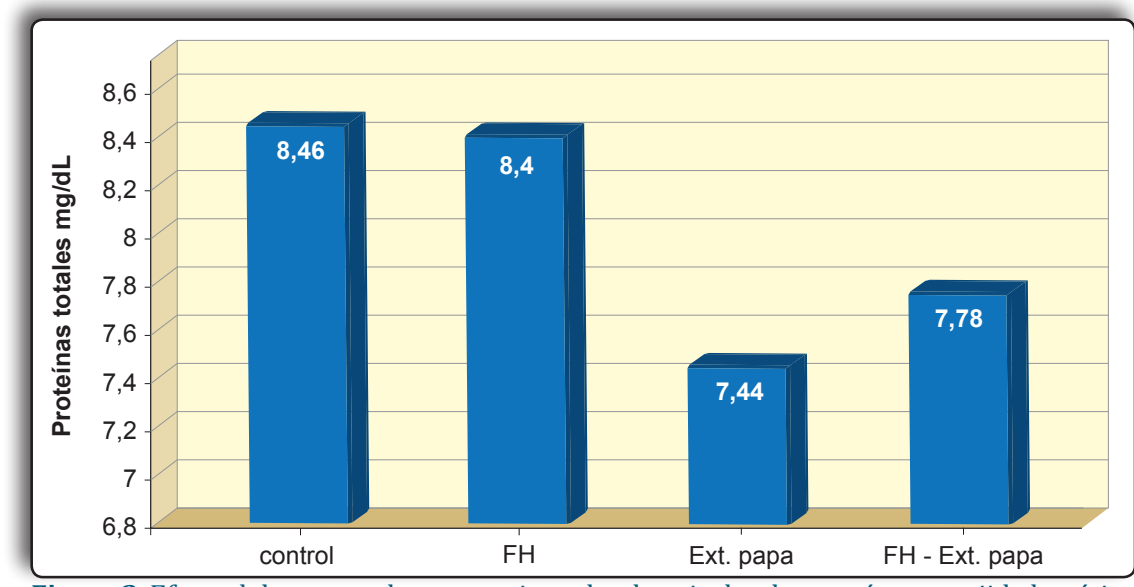

Figura 3. Efecto del extracto de papa nativa sobre los niveles de proteínas en tejido hepático de ratas en un modelo de hiperbilirrubinemia.

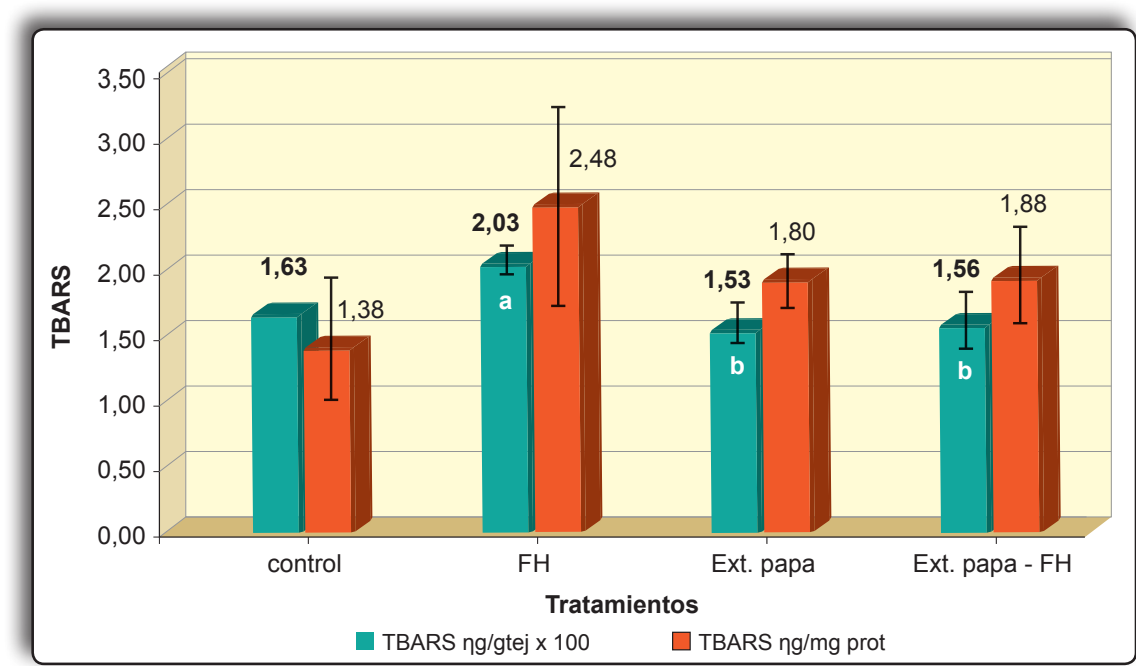

Figura 4. Efecto del Extracto de papa nativa en la Lipoperoxidación hepática en estado de hiperbilirrubinemia. Las diferencias significativas contra el control son expresadas como (a) y las diferencias contra $\mathrm{FH},(p<0,05)$.

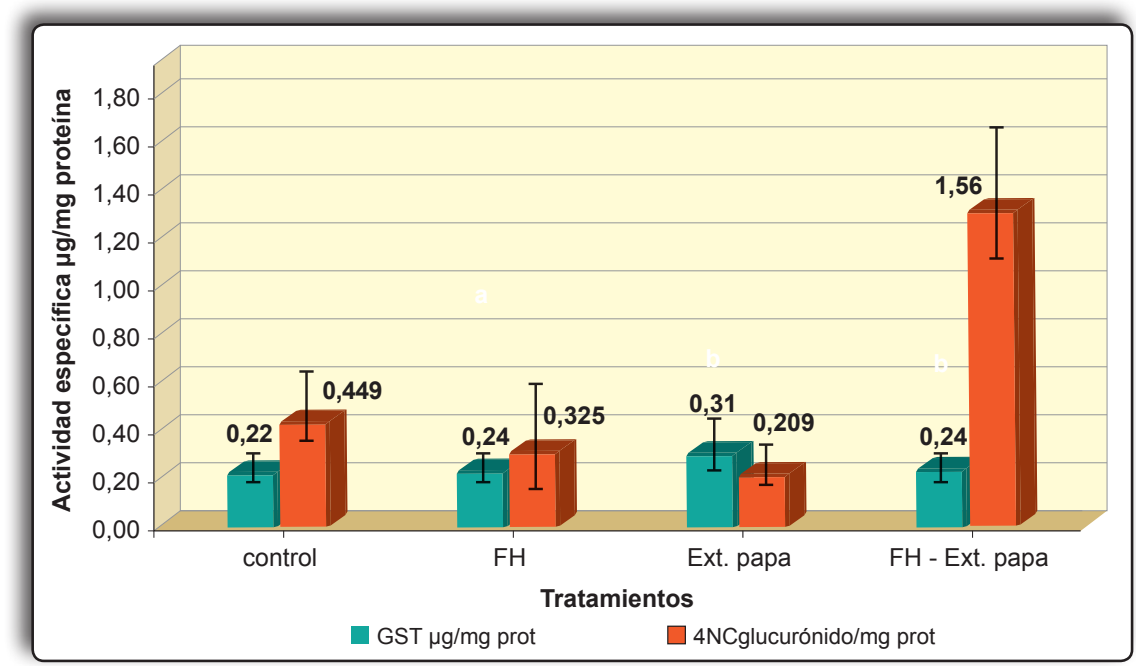

Figura 5. Efecto del Extracto de papa nativa sobre la actividad específica de las enzimas de detoxificación de Fase II en hiperbilirrubinemia inducida. Glutation S-transferasa (GST) y UDP-glucurónido transferasa (4NCglucurónido). temperatura ambiental entre 23 a $26^{\circ} \mathrm{C}$ y una humedad relativa de 60 a $70 \%$ con 12 horas de luz/oscuridad; fueron alimentadas ad libitum. Se realizó una distribución aleatoria en 4 grupos de siete individuos cada una, a los que se administró tratamiento durante 7 días:

Grupo I control: $1 \mathrm{~mL}$ de suero fisiológico por vía orogástrica durante siete días y o,2 $\mathrm{mL}$ de suero fisiológico vía intraperitoneal 48 horas antes del sacrificio.

Grupo II fenilhidrazina: $1 \mathrm{~mL}$ de suero fisiológico por vía orogástrica durante siete días y fenilhidrazina (disuelta en una mezcla hidroalcohólica 1:1) $60 \mathrm{mg} / \mathrm{kg}$ de peso corporal, por vía intraperitoneal 48 horas antes del sacrificio.

Grupo III extracto de papa: extracto acuoso de papa $665 \mathrm{mg} / \mathrm{kg}$ de peso corporal por vía orogástrica y $0,2 \mathrm{~mL}$ de suero fisiológico vía intraperitoneal 48 horas antes del sacrificio.

\section{Grupo IV fenilhidrazina-extracto} de papa: extracto de papa $665 \mathrm{mg} / \mathrm{kg}$ de peso corporal, por vía orogástrica durante 7 días y fenilhidrazina 60 $\mathrm{mg} / \mathrm{kg}$ de peso corporal por vía intraperitoneal 48 horas antes del sacrificio.

Al octavo día las ratas fueron anestesiadas con éter etílico y se procedióa extraersangreporpunción cardiaca, siendo inmediatamente sacrificadas para extraer el hígado y realizar los ensayos bioquímicos. La determinación de bilirrubina total se realizó empleando el protocolo del Laboratorio Bialex, para el hematocrito el método de Wintrobe y para las proteínas totales el método colorimétrico de Lowry ${ }^{(6)}$. Asimismo, el contenido de GSH total se determinó por el método de Ellman ${ }^{(7)}$, en el cual el GSH es oxidado por el ácido 5, 
5 '-ditiobis-2-nitrobenzoico (DTNB) y reducido por el nicotinadenindinucleotidofosfato reducido (NADPH) en presencia de glutatión reductasa. La formación de ácido 2-nitro-5-tiobenzoico (TNB) fue monitoreada a $412 \mathrm{gm}$. La actividad de la enzima GSH-transferasa se midió de acuerdo a la técnica espectrofotométrica descrita por Habig ${ }^{(8)}$. La mezcla de reacción contenía, en un volumen de $1 \mathrm{~mL}$, buffer fosfato $0,1 \mathrm{M} \mathrm{pH}=6,5$; 1-cloro-2,4-dinitrobenceno $1 \mathrm{mM}$; GSH 4mM; muestra problema $10 \mu \mathrm{g} / \mathrm{mL}$. La actividad se determinó midiendo la absorbancia del conjugado formado enzimáticamente a $340 \mathrm{\eta m}$, en forma continua durante $3 \mathrm{~min}$ a $20^{\circ} \mathrm{C}$. La actividad de la enzima UDPGT, se midió por el método de Letelier et al ${ }^{(9)}$, determinando el p-nitrofenol que queda después de 15 minutos de incubación en las siguientes condiciones: $0,5 \mathrm{mM}$ de p-nitrofenol; 2 mM de UDPGT; $100 \mathrm{mM}$ Tris $\mathrm{HCl} \mathrm{pH}=$ 8,$5 ; 4 \mathrm{mM} \mathrm{MgCl} ;$ y $2 \mathrm{mg} / \mathrm{ml}$ de proteína microsomal. La velocidad de reacción fue determinada en función de la formación de producto dependiente del tiempo y la concentración. Para determinar la peroxidación lipídica (MDA) se empleó el método colorimétrico de sustancias reactivas al ácido tiobarbitúrico (TBARS). El producto MDA se determinó por espectrofotometría a una longitud de onda de $532 \mathrm{gm}{ }^{(10)}$.

\section{DISCUSIÓN}

En la marcha fitoquímica se identificaron compuestos fenólicos, saponinas_y flavonoides, además de polifenoles, que también fueron reportados por Villacrez ${ }^{(11)}$ en estudios de papas nativas ecuatorianas. Los resultados muestran que

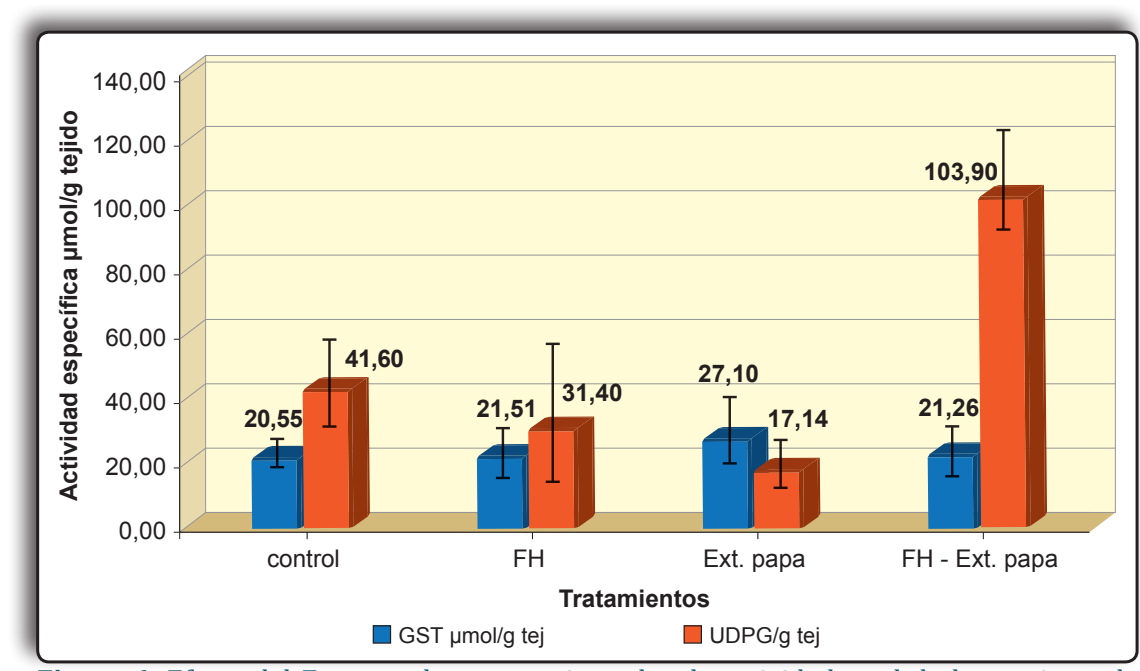

Figura 6. Efecto del Extracto de papa nativa sobre la actividad total de las enzimas de Detoxificación de Fase II en hiperbilirrubinemia inducida. Glutation S-transferasa (GST) y UDP-glucurónido transferasa (4NCglucurónido). los polifenoles y saponinas son los más abundantes en el extracto y serían responsables del efecto antioxidante y de la actividad sobre las enzimas de fase II del metabolismo ${ }^{(3)}$ (tabla 1). Es su contenido de antioxidantes, lo que permite incluir a la papa dentro de los alimentos funcionales ${ }^{(2)}$.

El modelo de hiperbilirrubinemia inducido por la anemia hemolítica, expresado por el hematocrito y los niveles de bilirrubina total sérica (figuras 1 y 2 ), muestran la efectividad de la fenilhidrazina a 6o mg/ $\mathrm{kg}$ de peso. En ambas figuras se observan diferencias significativas entre el grupo control y el grupo con inducción de hiperbilirrubinemia. Igualmente, puede observarse que el grupo al que se le administró el extracto de papa, conserva ambos parámetros a niveles comparables con el grupo control. Del mismo modo puede observarse que a nivel sérico ya se observa una tendencia a la recuperación de la bilirrubina total en el grupo IV, el resultado muestra una disminución de $25 \%$ con respecto al grupo III con hiperbilirrubinemia. Este resultado es importante porque indicaría la participación de metabolitos secundarios del extracto de papa en este grupo. Los probables mecanismos bioquímicos se podrían explicar con los resultados del contenido de GSH total, actividad de la UDP-glucuroniltransferasa y glutatión S-transferasa. En relación a las proteínas se observa una disminución de los niveles de proteínas totales en el grupo III y grupo IV respecto al grupo I y II (figura 3).

La formación de TBARS como expresión de lipoperoxidación, principalmente a nivel de membranas, fue significativamente mayor en el grupo II, que fue injuriado para hacer hiperbilirrubinemia (figura 4). En el mismo gráfico, el efecto antilipoperoxidación del extracto de papa (grupo III) puede observarse de manera significativa al compararlo con el grupo II. El grupo IV exhibe valores semejantes al grupo I y al grupo que sólo recibió el extracto (grupo III). Se explicaría porque a la dosis de extracto empleada, sus metabolitos secundarios antioxidantes, como los polifenoles, y los mecanismos antioxidantes a nivel hepático estarían controlando el estrés oxidativo.

Suárez et al. ${ }^{(10)}$, en el estudio "extracto acuoso de Lepidium 
meyenii Walp (maca) y su papel como adaptógeno, en un modelo animal de resistencia física", encontraron que el efecto protector del extracto en el marcador bioquímico de peroxidación lipídica TBARS, se producía a la dosis de 800 y $1200 \mathrm{mg}$ extracto/ $\mathrm{kg}$ de peso corporal, dosis superior a la empleada en nuestro estudio. Resultados protectores para la lipoperoxidación también fueron reportados por Toro et $a l^{(12)}$ en un modelo de hepatotoxicidad inducida por $\mathrm{CCl}_{4}$; igualmente con el mismo modelo, Mandal et al. ${ }^{(13)}$, empleando carnosol, un metabolito fenólico de Physalis peruviana, a una dosis de $250 \mathrm{mg} / \mathrm{kg}$ de peso. Es importante resaltar que el estudio está basado en un extracto, lo que puede llevar a proponer que algunos de los metabolitos fenólicos del extracto de papa también podrían exhibir semejante o mejor comportamiento.

La actividad específica de la UDPglucuroniltransferasa aumentó significativamente $(p<0,01)$ en el grupo con hiperbilirrubinemia que recibió, además, el extracto frente a los otros tres grupos; el mismo resultado se observa cuando la actividad es expresada en actividad total y específica (figuras 5 y 6). Sin embargo, en ambas figuras puede observarse que el resultado de la actividad específica y total de la glutatión S-transferasa no exhibe ninguna diferencia significativa en los cuatro grupos.

La UDP-glucuroniltransferasa tiene un rol importante en la eliminación de una serie de sustancias endógenas, especialmente la bilirrubina producto de la degradación de hemoglobina. La reacción del donador ácido UDP-glucurónico se produce por conjugación con grupos funcionales como hidroxilo, carboxilo, amino o tiol de una molécula blanco activada (aglicona) formándose $\mathrm{O}-, \mathrm{N}-$, o S-glucurónidos, respectivamente. La enzima forma un $\alpha$-D-glucurónido, que produce un incremento de la polaridad de la aglicona de manera que puede ser fácilmente excretada por la bilis o la orina ${ }^{(14)}$. En el presente estudio se observa que el extracto de papa incrementa de manera significativa la actividad de esta enzima, pero al ser esta una investigación inicial no permite todavía expresar el mecanismo de activación.

En el caso de la glutation S-transferasa, se trata de un grupo importante de proteínas halladas en dos grandes superfamilias, la más abundante es la citosólica o soluble, pudiendo constituir hasta el 10\% de las proteínas solubles del hepatocito. Aún con estas características, el inductor de la hiperbilirrubinemia empleado en este estudio, no es sustrato para esta enzima, razón por la que no se ha hallado diferencias significativas en los cuatro grupos. Sin embargo, puede notarse que el grupo tratado sólo con extracto de papa exhibe un ligero incremento de la actividad enzimática ( $40 \%$ expresado como actividad específica y $32 \%$ expresado como actividad total) con respecto al control. Esta enzima además cumple otras funciones bioquímicas diferentes a la conjugación ${ }^{(15)}$ como la actividad antioxidante.

\section{CONCLUSIONES}

El extracto acuoso de papa nativa "puca simi” contiene compuestos fenólicos, saponinas y flavonoides.

El extracto de papa nativa "puca simi" incrementa de manera significativa la actividad de la UDP-glucuroniltransferasa.

\section{REFERENCIAS BIBLIOGRÁFICAS}

1. Coultate P. Alimentos, química de sus componentes: pigmentos. Acribia. Zaragoza, 1984. p. 104-28.

2. Suárez S, Trabucco J, Oré R, Arnao I, Rojas L, Ramírez J. Potencial antioxidante de 62 variedades de papas nativas del sur andino del Perú. An Fac med 2012; 73 Sup 1: 36 .

3. Stevenson D, Hurst R. Polyphenolic phytochemicals just antioxidant or much more?. Cell Mol Life Sci 2007; 64(22): 2900-16.

4. Bustíos C, Dávalos M, Román R, Zumaeta E. Características epidemiológicas y clínicas de la cirrosis hepática en la Unidad de Hígado del HNERM EsSalud. Rev Gastroenterol Perú. 2007; 27(3):238-45.

5. Vargas N. Efecto hepatoprotector y antioxidante del extracto y los principios activos de Geranium schiedeanum. [Tesis que para obtener el grado de: Maestra en ciencias biomédicas y de la salud]. Universidad Autónoma del Estado de Hidalgo. HidalgoMéxico, 2012.

6. Lowry OH, Rosebrough NJ, Farr y AL, Randall RJ. Protein measurement with the folin phenol reagent. Biol Chem 1951. 193: 265-75.

7. Ellman G. Tissue sulfhydryl groups. Arch Biochem Biophys 1959; 82(1): 70-7.

8. Habig W, Pabst M, Jakoby W. Glutathione S-Transferases. The first enzymatic step in mercapturic acid formation. J.Biol Chem 1974; 249(22): 7130-9.

9. Letelier M, Del Villar E, Sánchez E. Drug tolerance and detoxicating enzymes in Octodon degus and Wistar rats. A comparative study. Comp Biochem Physiol 1985; 8oㅡ (1), 195-98.

10. Suárez S, Oré R, Arnao I, Rojas L, Trabucco J. Extracto acuoso de Lepidium meyenii Walp (maca) y su papel como adaptógeno, en un modelo animal de resistencia física. An Fac Med 2009; 70(3): 181-5. 
11. Villacrés E, Quilca N, Muñoz R, Monteros C, Reinoso I. Caracterización morfológica, física, organoléptica, química y funcional de papas nativas (Solanum ssp.), para orientar sus usos. I Congreso Internacional de Investigación y Desarrollo de Papas Nativas-PAPANAT. Ecuador, 2010.

12. Toro RM, Aragón DM, Ospina LF. Efecto hepatoprotector de un extracto de cálices de Physalis peruviana en hepatotoxicidad inducida por $\mathrm{CCl}_{4}$ en ratas wistar. Vitae 2013; $20(2)$ : 125-32.

13. Mandal AK, Das N. Sugar coated liposomal flavonoid: a unique formulation in combating carbontetrachloride induced hepatic oxidative damage. Journal of Drug Targeting 2005, 13(5): 305-315.

14. Kurkela M, Patana AS, Mackenzie PI, Court MH, Tate CG, Hirvonen J, et al. Interactions with other human UDP- glucuronosyltransferases attenuate the consequences of the $\mathrm{Y}_{4} 85 \mathrm{D}$ mutation on the activity and substrate affinity of UGT1A6. Pharmacogenetics and Genomics 2007; 17 (2): 115-26.

15. Sherratt P, Hayes J. Glutatione S-transferases. University Dundee. UK, 2001.

Manuscrito recibido el: 15/o8/14

Aceptado para su publicación el: 04/09/2014

\section{Correspondencia:}

Nombre: $\quad$ Emilio Ramírez Roca

Dirección: Calle Droseras 134, Salamanca-Ate. Email:_emilioramirezroca@hotmal.com 